\title{
Principles And Problems Underlying Testing The Effectiveness Of Blast Protective Footwear
}

\author{
EJ Chaloner, J McMaster, DE Hinsley
}

\begin{abstract}
Anti-personnel landmines are a continuing threat to soldiers and civilians working overseas in post conflict situations. Several groups of governmental and commercial scientists are currently designing and/or testing footwear to protect the lower leg from the effects of close proximity blast. The general principles surrounding testing of protective footwear are examined together with an assessment of the known progress to date and the strengths and weaknesses of the designs produced.
\end{abstract}

Keywords: Anti-personnel landmine; protection; military surgery; war; trauma.

\section{Introduction}

The global problem with uncleared antipersonnel mines is well known (1-4). Despite a recent international treaty to ban these weapons, there are still significant numbers deployed worldwide, and their use is likely to remain a feature of conflicts for years to come. The commonest injury is caused when a person detonates a buried blast mine by treading on its pressure plate with the foot. This is known as a Pattern 1 injury (1) according to the International Committee of the Red Cross classification. The explosion results in a traumatic amputation of part of the detonating limb with fragmentation damage to the contralateral limb.

The mechanism of Pattern 1 antipersonnel mine injury has been recognised for many years, and several attempts have been made to design effective footwear to protect against detonation of an explosive charge under the foot.

The majority of these designs relied on a combination of ballistic composites such as Kevlar (Dupont Corp, USA), honeycombed lightweight metals to absorb or deflect blast, or a standoff to distance the foot from the explosion. Few of these designs were taken forward into widespread use due to the inability of the designers to produce a boot which had both a reasonable level of protection and which was functionally compatible with wear in the field.

As a consequence of the large number of mine clearance operations now in progress around the world, and the increased number of peacekeeping deployments involving NATO troops, the issue of blast protective footwear has once again become the focus of attention. Several new products have been developed and marketed as offering the solution to the threat posed by buried blast mines. The majority incorporate aspects of the previous design strategies although a number of new ideas have been developed. These include the Aigis boot which incorporates a blast attenuating substance known as TABRE along with a Dyneema layer to keep out fragments, the Welco boot from the USA, using honeycombed metal as a blast attenuator along with a $\mathrm{V}$ shaped sole in an attempt to deflect some of the blast energy and a 'spider boot' developed by MedEng of Canada which uses four pods on each corner of the boot to reduce the risk of mine detonation and enhance stand - off.

Some of the newer designs are aimed at the infantry soldier with the intention that he would be able to wear the blast protective boot as normal combat footwear, while strapping on an overshoe in areas of enhanced mine threat (Wellco Boot, USA), whilst the Aigis and MedEng footwear is designed for the niche market of specialist mine clearance and engineer survey work.

Regrettably, many of the designs on the market have not been tested adequately for clinical effectiveness. Much product literature mistakenly equates resilience of the boot construction with protection of the foot within. It is quite possible for a boot to remain structurally intact following an explosion whilst the foot within is so damaged as to be unsalvageable by surgery. The methodology of the experiments by which testing of several boots has been conducted is open to criticism and few programmes have been conducted in a way which allows meaningful comparison of results between different groups. Adequate assessment is also hindered by publication bias driven by commercial considerations and issues of national security.

There is a need for consensus to develop on the principles involved in testing the effectiveness of such mine protective footwear in order for different groups trial methodologies to be harmonised, permitting valid comparisons to be drawn between experiments. Elimination of some of the preconceptions in the mine clearance community about the nature of the interaction between an anti - personnel mine and the lower limb, and the important issues involved in protection would be a useful by product.
Porton Down,

Salisbury. 
In this paper we highlight the clinical and engineering issues involved in the pathophysiology of close proximity blast injury to the lower limb, and the important aspects involved in testing the various designs of protective footwear.

\section{Pathophysiology}

The clinical manifestations of blast injury to the unprotected limb have been well documented (1). The blast wave causes a traumatic amputation of part of the foot and lower leg with stripping of the soft tissues from the underlying bone for a variable distance further up the leg. Soil and other debris is often propelled high into the leg along the tissue planes, and burning, due to the hot gases of detonation, further injures the soft tissues. Although there has been surprisingly little basic research done into the mechanics of anti - personnel mine blast injury, a great deal of clinical experience in this area has been published. Some authors have attempted to salvage limbs exposed to unattenuated close proximity blast (6-9) with variable results. However, the vast majority of limbs exposed to anti-personnel mine blast require a major formal amputation. The level of the amputation is determined by the degree of soft tissue damage and contamination rather than by the level of bony injury, because the skin and muscle of the limb is usually damaged proximal to the level of bone fracture.

Successful protection of the lower limb can only be achieved by reducing the energy transmission into the leg to a level at which the soft tissue and bony structures can be salvaged, either by natural recovery processes, or with reconstructive surgery. Furthermore, simple limb salvage alone does not necessarily imply a satisfactory clinical outcome. If the salvaged limb is consistently painful, or lacks an adequate nerve supply, the patient may be better served by an amputation and mobilisation on a prosthetic limb. If amputation is required, rehabilitation is much more successful if the knee joint is preserved, due to the much lower energy requirements of walking on a below knee prosthesis and the added benefits of proprioreception offered by the intact knee joint.

Despite the recent advances in boot design, it is highly unlikely that footwear can be produced that will completely protect the foot from all injury resulting from a close proximity anti - personnel mine blast. The phrase 'defeating the landmine' should probably be expunged from the vocabulary - anyone standing on an anti - personnel mine will sustain a significant limb injury, regardless of the size of the charge or the type of footwear worn. The challenge is whether that injury can be reduced to one from which the individual can make a good functional recovery rather than face an inevitable amputation.

\section{Assessing Protection}

Realistically modelling the effects of protective systems on the live human leg is extremely difficult to achieve with a high degree of biofidelity (the extent to which a given injury to the model reflects an injury to human tissue). The goal therefore is to devise a satisfactory model, which will accurately simulate the effect of close proximity blast on the lower limb of a young healthy individual and from the information gleaned to be able to predict the likely clinical outcome. The options that have been utilised in descending order of biofidelity (in terms of soft tissue injury and prediction of outcome) are:

Whole Cadavers

Amputated Human limbs

Synthetic Limb Preparations/Animal

Models

Metal Rigs

Computer Modelling

\section{Human Limb Specimens}

Human tissue specimens provide the most accurate information on the effects of blast on the complicated bony structures of the foot/ankle complex and offer some information on injury to the soft tissues. Whole cadavers offer the opportunity to study the effect of the transmission of the blast wave higher up the leg, particularly on the knee and the hip joint, whereas isolated limb specimens are limited to the foot, ankle and lower leg area (Figure 1). Whole cadavers also permit movement of the

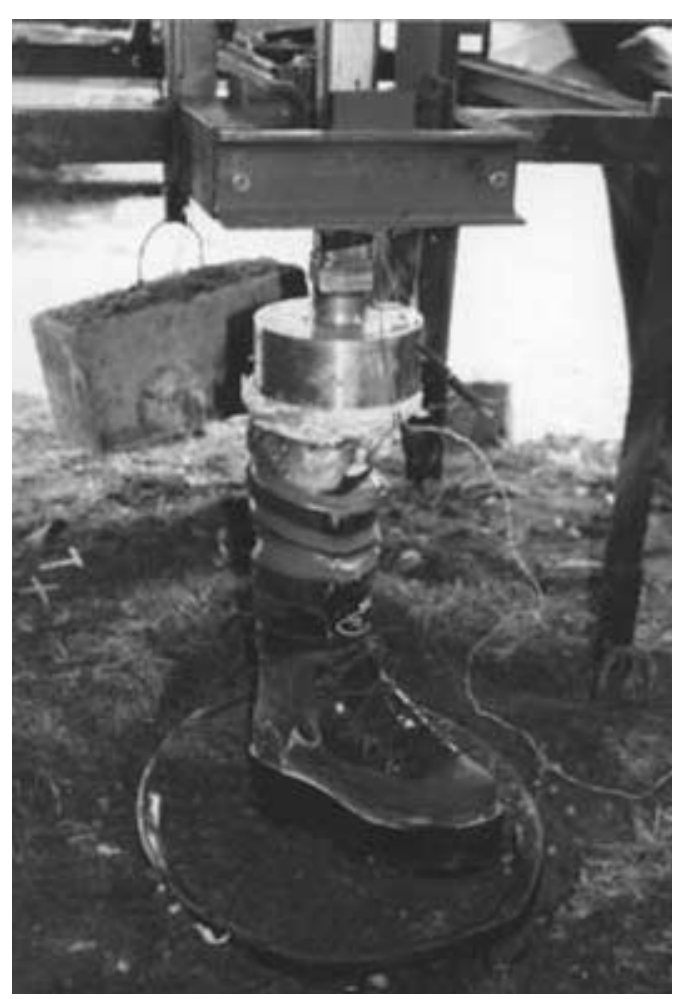

Fig 1. Amputated human lower limb model. 


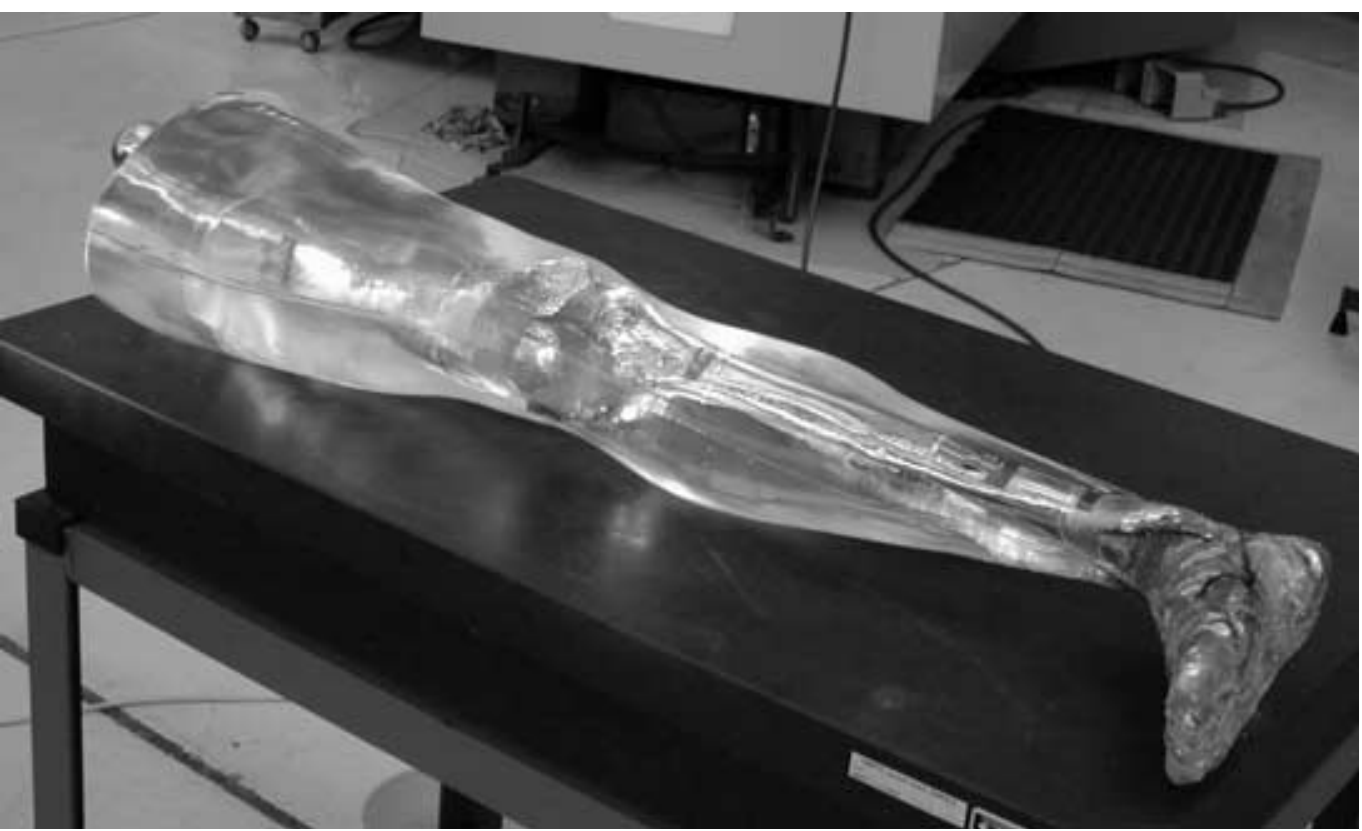

Fig 2. The Frangible Synthetic Leg (FSL). (Courtesy of the Defence Science and Technology Organisation, Australia).

preparation in several planes, rather than being constrained to one plane by being fixed in a rig in the case of single limb experiments.

The main drawbacks of human tissue work are the ethical and health and safety aspects, which need to be considered when conducting any experiments involving biological samples. Furthermore, there is considerable variability in specimens in any biological material. Amputated limbs are removed as a consequence of disease (usually end stage peripheral vascular disease) and are therefore from an elderly population. The density of the bones in these patients is considerably lower that that of a younger group, and therefore the bones are more susceptible to fracture at a given load. Soft tissue in this group of patients has often been compromised by the effects of chronic ischaemia and may be more vulnerable to the effects of post mortem blast injury. Assessment of injury patterns in any biological system is also open to a degree of subjective interpretation and, as the material is dead tissue, the extrapolation of a given observed injury into a clinical outcome is clearly a matter of opinion, rather than observable fact.

\section{Synthetic Limbs}

A variety of synthetic limb preparations have been used in blast protection experiments, ranging from crude simulations of the foot with rubber or wooden blocks, to highly sophisticated surrogates incorporating ballistic gelatine as a human soft tissue simulant.

The cruder synthetic models have little value other than in assessing the basic structural integrity of the protective layer in initial studies. There is little correlation between a crack in a wooden block following exposure to a given amount of blast energy and the effects on a living foot.

The more sophisticated artificial limbs such as the Australian Frangible Synthetic Leg (Figure 2) have certain advantages over human tissue. They circumvent the ethical and health and safety aspects surrounding human tissue work and some of the variability of human limb specimens by modelling the surrogate bone on data pertaining to the young age group that are likely to be injured in mine clearance operations.

The validity of such surrogates in simulating the effects of blast on soft tissue is in question. The soft tissue is simulated by ballistic gelatine, which has been validated as a reasonable model for ballistic trauma in terms of energy transfer (10), but not for the more complex blast injury. Furthermore, a satisfactory simulation of skin on these models is difficult to achieve. That being said, a series of recent tests comparing results of tests done on the Frangible Limb with the human cadaver work from the LEAP programme in the United States has produced good correlation between the two (11). The main problem with the frangible surrogate is in modelling the bones of the hindfoot. In terms of bony injury, damage to the calcaneum, talus and the ankle joint is the most important determinant of the salvage potential of the leg, assuming the soft tissues are viable. Paradoxically, advanced surrogate limbs are extremely expensive to obtain, far more so than human legs (which are usually donated free by patients who wish to donate tissue for medical experimentation).

\section{Animal Specimens}

The red deer tibia appears to be very similar in structural integrity to the human bone and 


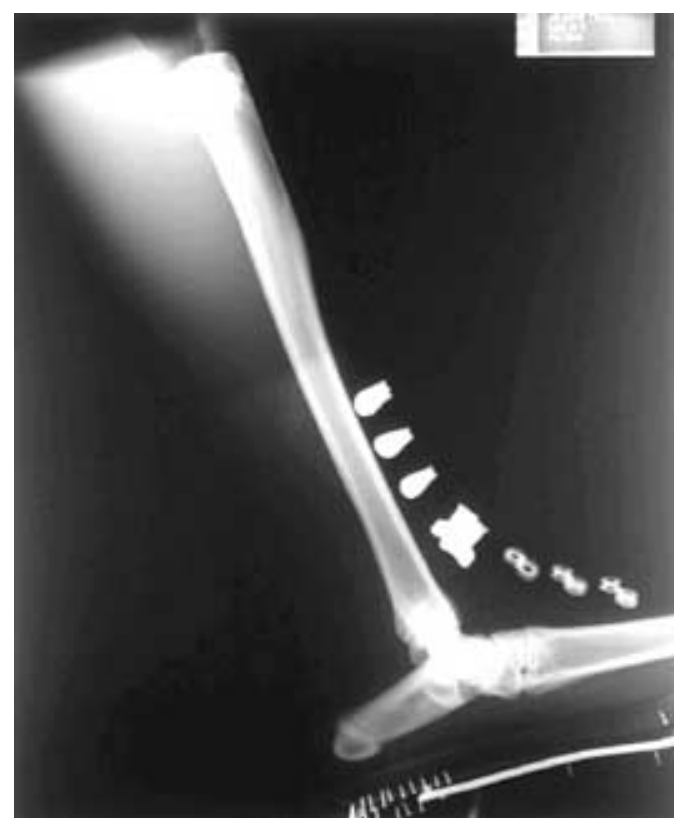

Fig 3. Red deer lower limb within a combat boot.

has been used as a lower limb model for blast protection experiments (Figure 3) (12). In common with synthetic limbs, use of tissue recovered from dead animals is not subject to ethical constraints or health and safety issues associated with using human tissue. In addition, they are cheap, relatively easy to obtain and have the advantage of good quality bone from young animals. Historically, quadrupeds such as pigs, sheep and dogs have been used for blast experimentation. They all walk on their "toes" due to inherent anatomical differences, consequently the calcaneus does not have to withstand axial loading. Although the talus does share similarities with its human counterpart, the midfoot and forefoot is simplified, possessing fewer tarsal and metatarsal bones.

Anti - personnel blast injury rarely results in fracture to the tibia without severe bony damage to the foot/ankle complex. Similarly, the soft tissue configuration of the human lower limb is unique to bipeds. It is the soft tissues of the leg, injured by stripping, contamination and burn injury that result in high amputations. Anatomical differences and the shortcomings of gelatine as a soft tissue surrogate may limit the usefulness of these models.

\section{Metal Limb Rigs}

Metal limb rigs are of some value in the early phases of footwear development. Tests with such rigs can confirm the tolerance limits of

Table 1. Accelerations observed in various protective boot experiments measured in ' $g$ '

\begin{tabular}{|c|c|c|c|c|}
\hline & \multicolumn{2}{|c|}{ Chaloner et al 2001 (15) } & \multicolumn{2}{c|}{ Makris 2000 (13) } \\
\hline Charge of C4 & Combat Boot & Aigis Boot & Welco Boot & 'Spider' Boot \\
\hline 25 & & 565 & 10,300 & 150 \\
\hline 50 & 863 & 388 & & 685 \\
\hline 100 & & & & 1315 \\
\hline
\end{tabular}

a variety of boot designs, narrowing down the number of boots that need to be tested by more expensive and complex means. Basic instrumentation data can be gleaned from metal rig trials, which is helpful in investigating the general principles of energy attenuation, although gathering load data from the metal shaft can be compromised by 'ringing' of the blast wave.

If appropriate biomechanical indices of limb injury can be calculated, metal rigs do provide a cheap way of performing "what-if" experiments altering individual parameters, for example what biomechanical benefit does altering stand-off, alone, afford?

\section{Computer Modelling}

A well validated computer modelling system would be of tremendous value, both in the design and testing phases of blast protective clothing. Unfortunately, such a model does not exist at the present time. One of the difficulties is that too few human limb tests have been conducted to glean the basic data required to develop models that are both biomechanically and anatomically accurate.

\section{Instrumentation}

A number of parameters can be measured in blast experiments and used to compare results within a series of tests by the same workers and between groups doing similar studies. Parameters, which have been measured in the published tests to date, include load, acceleration, strain on the tibia, vertical displacement and pressure within the protective footwear during the blast event.

Use of these data can give a comparison between experiments done on blast related energy (which are small in number), and the large database available from the automotive industry. Instrumentation gives a quantifiable assessment of the forces acting on the test specimen which are otherwise open to the vagaries of observer interpretation. However, there is no agreement on which modalities are important to measure, nor on the implications of instrumentation for clinical outcome. Data from instrumentation can show surprising variability even within the same series of experiments and comparisons between different groups can be difficult to make (see Table 1).

The measurement techniques were essentially the same in the two groups (Kistler 3 way accelerometers), although experimental design did differ. The British group used isolated human legs constrained to permit only uniaxial motion, while the Canadians used the Frangible Human Limb surrogate held vertically by four guy ropes. These differences in methodology probably do not explain such enormous variation in results.

Similarly, the loads measured with similar charge sizes can vary significantly depending 
Table 2. Load recorded through Tibia in kn (Chaloner et al 2001 (15), Harris et al 1999 (14)).

\begin{tabular}{|c|l|c|c|c|c|}
\hline \multicolumn{2}{|l|}{ Charge size and type } \\
\hline & Boot Type & $\begin{array}{c}\text { 29gms Tetryl } \\
(\mathbf{M 1 4} \text { mine) }\end{array}$ & $\begin{array}{c}\text { 25gms } \\
\text { C4 }\end{array}$ & $\begin{array}{c}\text { 50gms } \\
\text { C4 }\end{array}$ & $\begin{array}{c}\mathbf{7 5 g m s} \\
\text { C4 }\end{array}$ \\
\hline UK & Combat Boot UK & & & 8.16 & \\
\hline & Aigis Boot & & 17.50 & 13.74 & 20.13 \\
\hline US & $\begin{array}{l}\text { Combat Boot and Aigis } \\
\text { Overboot }\end{array}$ & & 18.73 & 17.80 & \\
\hline & Combat Boot US & 7.13 & & & \\
\hline & Welco Boot & 10.01 & & & \\
\hline
\end{tabular}

on experimental design. Table 2 shows the loads recorded by the UK Aigis Group and the US Institute of Surgical Research Group San Antonio in a series of broadly similar experiments (14). The difference in the design was that the UK group used amputated human limbs with axial loads of $67 \mathrm{Kg}$ constrained to move in only one plane (vertically). The US group using whole cadavers had multiplanar movement with minimal axial loading on the specimen, which may be why their peak loads are significantly lower for similar charge sizes. The peak loads are the result of blast attenuation by both the boot and the structures of the leg. This is of particular significance in those legs where gross disruption of the tissues has occurred. Legs that were severely damaged recorded lower peak loads from cells placed in the tibiae, presumably due to absorption of energy during the destruction of distal structures.

There is considerable debate about the value of any instrumentation in the testing of blast protective footwear as some investigators feel that its contribution is minimal compared to the all important clinical effects on the bone and soft tissue. Furthermore given that the time to peak loading seen in automotive tests is of the order of 20 milliseconds, and the peak load time in a blast event is approximately 2 milliseconds, some argue that comparisons between the two sets of data are meaningless. In automotive related injury tolerance, testing the peak load will often represent the load at which there is structural failure of the leg. In the mine boot tests carried out by the Aigis group, failure of the limbs used would have occurred before the peak loads recorded in typical automotive testing were achieved. We believe this may be due to the higher rate of loading in the blast event.

Although it is possible that blast energy will inflict more damage on the small blood vessels and nerves of the leg than car crash energy transfer, measurement of the various physical effects should continue to be a feature of such experiments in order to move towards a standard methodology for testing. This would help in comparing data between groups, and in quantifying the physical effects of the various protective systems. A consensus view is needed on which parameters are important and how they should be measured.

\section{Experimental Design}

Experimental design is a crucial factor in obtaining methods of standardisation. Variables in design complicate an already complex system even further and lead to needless argument on minor points about whose experiment is 'more valid'. At present, there are three groups in the UK, the USA and Canada who have conducted and presented well designed trials.

At present the main variables (other than boot design) in the published experiments include the type of charge used, the type of soil the charge is in and the depth to which it is buried, the degree to which the limb is preloaded and finally the position of the charge in relation to the foot.

The main issue surrounding the type of charge relates to the standardisation of charges within anti - personnel landmines. The majority of mines use a TNT / RDX main charge. Some mines may fail to sustain a high order detonation due to poor quality control, climactic conditions or the time that the mine has spent in the ground. For reasons of availability, as well as for the advantages of malleability and accurate measurement, many groups choose to use PE4 or C4 plastic explosive as a surrogate mine. There is some disagreement as to what extent surrogate mines mimic the explosion of a true landmine. The position of the detonator within the charge mass may substantially alter the progression of the blast wave and therefore it's interaction with the foot above. This may be the reason for some of the discrepancy in recorded loads in the Aigis Group's experiments on metal and human legs. The detonator was placed at the side of the charge in the metal limb tests and directly in the middle of the charge in the case of the human limb tests.

The use of PE4/C4 is likely to provide more explosive force on a weight equivalent basis than a TNT/RDX mixture. PE4/C4 consists of RDX (cyclotrimetheylenetrinitramine) combined with a plasticiser. It has a greater brisance (the rapidity with which an explosive develops its maximum pressure) than TNT alone (16).

The density and moisture content of soil also affects the energy transfer between the mine and the foot. Heavy wet soil results in a greater energy transfer, whereas dry sand tends to dissipate the blast and reduce transfer into the limb. As the degree of injury is proportional to amount of energy transfer and its rate, this clearly has a bearing on the outcome of the experiment for any given charge size. Following from this is the issue of stand - off between the mine and the foot, which also has an effect on energy transfer. 
Recent experiments performed in Canada have shown that there is more energy transfer from a mine buried to a depth of 5 $\mathrm{cm}$ under soil than from one which is surface buried, due to increased momentum transfer from energised soil causing a more focused impact as opposed to the rapid dissipation of blast in free air $(17,18)$.

Finally, the location of the charge with respect to the foot has important implications for the pattern of injury sustained. Most experiments have been conducted with the charge placed under the heel of the human or surrogate limb to enhance comparisons within an experimental series and to produce the 'worst case' injury. However, the effect of 'off axis' detonation cannot be dismissed. It is equally likely in the real life situation that the mine will detonate under the forefoot, or to the side of the foot. Indeed, in the Med Eng design (13), off axis detonation is actually invited by the nature of the boot. Whilst off axis detonation may lessen the extent of injury to the bones and soft tissue of the hind foot, the rotational force applied to the tibia may result in fractures to the lower leg. In the off axis test conducted by the Aigis Group, the human limb sustained a compound fracture of the lower third of the tibia, which, although not directly limb threatening is certainly a significant injury which is challenging to treat.

\section{Conclusions}

Testing the effectiveness of blast protective footwear is a more complicated issue than has hitherto been appreciated by the demining and military communities. The design of experiments reported to date has thrown up as many questions as they have answered. There is considerable debate amongst the interested medical and engineering communities about which factors are important in determining the effectiveness or otherwise of a particular product. There is clearly a need for some standardisation of methodology to allow comparison between groups and to establish a benchmark of efficacy. This would serve to eliminate some of the extreme claims made by certain companies on behalf of products that have been inadequately tested. These issues are presently the subject of a NATO Technical Group comprising scientists and clinicians from several participant countries. The group is working towards establishing a standardised set of guidelines for development and testing of personal protective equipment against anti-personnel mine blast and is due to report in 2003 .

\section{References}

1. Coupland $\mathrm{R}$, Korver A. Injuries caused by antipersonnel mines: the experience of the International Committee of the Red Cross. $\mathrm{Br} \mathcal{F}$ Med 1991; 303: 1509-12.

2. Stover et al. The Medical and social consequences of land mines in Cambodia. $\mathcal{F} A M A$ 1994; 272: 331-6.

3. Ascerio A, Biellik R, Epstein A et al. Deaths and injuries caused by land mines in Mozambique. Lancet 1995; 346:721-4.

4. Chaloner EJ, Mannion SJ. Anti - personnel landmines: the Global Epidemic. Ann R Coll Surg Engl; 1995: 77: 1- 4.

5. Chaloner E J, The incidence of landmine injuries in Kuito, Angola. $\mathcal{F} R$ Coll Surg Edinb 1996: 41: 398-400.

6. McHale KA, Jackson JP. Reconstruction of orthopaedic War injuries - The medical centre experience. In Brown PW): Orthopaedic Lessons from recent wars. Techniques in Orthopaedics Phila., Lippincott - Raven Publishers 1995; 10: 211-221.

7. McHale KA, Pollock P. Limb salvage and reconstruction after blast injuries. The contributions of the methods of Ilizarov. Ann Med Milit Belg 2000; 14: 25-30.

8. Gur E, Atesalp S, Basbozkurt M, Atdogan N, Erler K. Treatment of complex calcaneal fractures with bony defects from landmine blast injuries with a circular external fixator. Foot and Ankle International; 1999; 20: 37-41.

9. Selmanpakoglu $N$, Guler $M$, Sengezer $M$, Turegun M, Isik S, Demirogullari $M$. Reconstruction of foot defects due to mine explosion using muscle flaps. Microsurgery 1998; 18: $182-8$.

10. Watkins FP, Pearce BP, Stainer MC. Assessment of Terminal effects of high velocity projectiles using tissue simulants. Acta Chir Scand Suppl 508: 1982 .

11. Bergeron D, Coley GG, Rountree MS, Anderson I, Harris RM. Assessment of foot protection against anti-personnel landmine blast using a frangible surrogate leg UXO April 2001.

12. Skelton C, Cannon L, Trimble K. Development of a Lower Limb Physical Model to assess the severity of Land Mine injury. XXIII International Congress on Military Medicine Jun 2000185.

13. Makris A, Islam S. Performance Tests of 'spider boot' for demining. World EOD Gazette Jan 2000; $33-44$.

14. Harris RM, Griffin L, Hayadar, Rountree MS, Bryant R, Rossitor N, Mannion SJ. Change in Lower Extremity Morbidity from Landmine Injury: an analysis of New Protective Footwear. In Orthopaedic Trauma Association, 15th Annual Meeting 1999 Charlotte N. Carolina USA.

15. Chaloner EJ, Holland SJ, McMaster J, Croft RJ. Unpublished results.

16. Iremonger MJ, Physics of detonations and Blast waves in The Scientific Foundations of Trauma Ed. Cooper GJ et al Butterworth Heinemann 1997 Oxford.

17. Bergeron D, Walker J, Coffey C. Detonation of $100 \mathrm{~g}$ Anti-personnel mine surrogate in sand: a test case for computer code validation. SR $668 \mathrm{Nov}$ 1998.

18. Bergeron D, Tremblay J. Canadian Research to characterise mine blast output MABS Oxford 2001 . 